\title{
Canadian Institute of Forestry $\quad-\quad$ Institut Forestier du Canada
}

\section{National Officers 1990-91 Bureau de direction national}

$\begin{array}{ll}\text { Gairns, H. } & \text { President } \\ \text { Whidden, A. } & \text { 1st Vice-President } \\ \text { Rotherham, A. } & \text { 2nd Vice-President } \\ \text { Ackhurst, P.W. } & \text { Past President } \\ \text { Cayford, J.H. } & \text { Editor } \\ \text { Burgess, D. } & \text { Editor } \\ \text { Fenton, T. } & \text { Production Manager } \\ \text { Lee, Chris } & \text { Executive Director }\end{array}$

15955 th Avenue, Suite \#101
P.O. Box 862 . Station "M"
19 th FI 1155 Metcalfe (Sun Life)
3950 Sunset Blvd.
25 Bernett's Grove Circle
R.R. \#1
Suite 1005, 151 Slater St.
Suite 1005, 151 Slater St.

$\begin{array}{ll}\text { Prince George, BC } & \text { V2L } 3 L 9 \\ \text { Halifax, NS } & \text { B3J 2V2 } \\ \text { Montreal, PQ } & \text { H3B 4T6 } \\ \text { N. Vancouver, BC } & \text { V7R } 3 \times 9 \\ \text { Nepean, ON } & \text { K2J 1W1 } \\ \text { Beachburg, ON } & \text { KOJ } 5 \mathrm{H} 3 \\ \text { Ottawa, ON } & \text { K1P } 5 \mathrm{H} 3 \\ \text { Ottawa, ON } & \text { K1P } 5 \mathrm{H} 3\end{array}$

Home

(604) $564-5978$

(514) $481-5818$

(604) $385-6935$

$613)$
$582-7129$
$691-1665$

(613) 591-1665

Business
$(604) 564-4115$
$(902)) 424-5703$
$(514) 866-6621$
$(604) 387-1191$
$(613) 589-2880$
$(613) 234-2242$
$(613) 234-2242$
$(613) 234-2242$

Fax

(604) $563-9679$

(902) $424-7735$
514) $866-4863$

(604) $387-1467$

(613) $589-2275$

(613) 234-6181

$(613)$
(613) $234-6181$
$234-6181$

\section{Section Officers 1990-91 / Responsables régionaux}

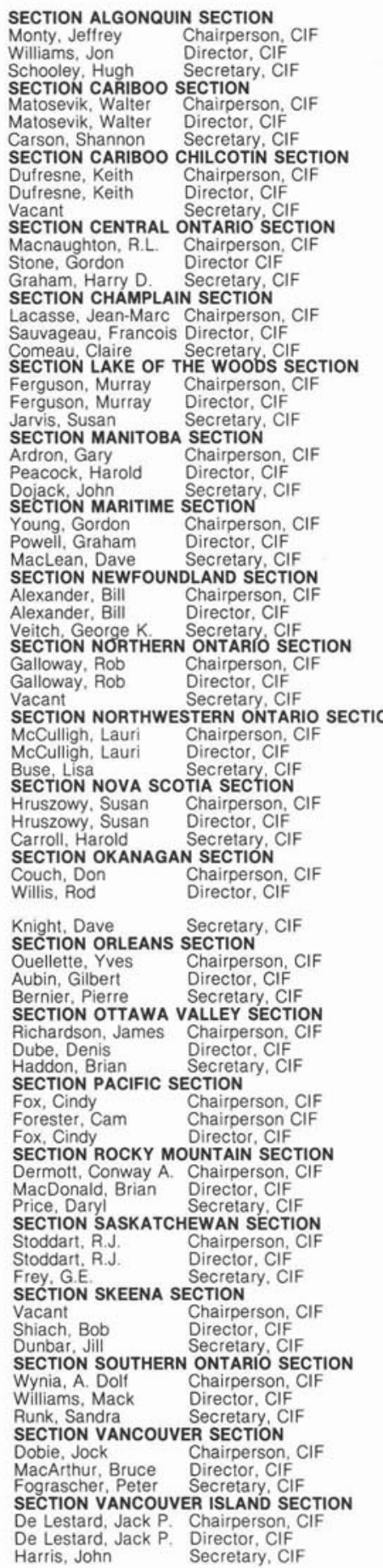

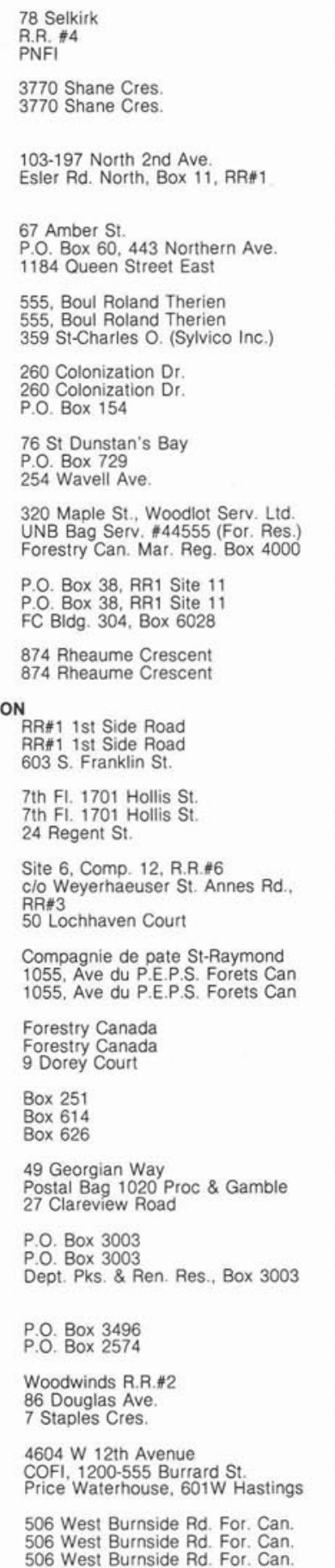

Petawawa, ON Petawawa. ON

Chalk River, ON

Prince George, BC

Prince George, BC

Williams Lake, BC V2G 1 Z5

Sault Ste. Marie, ON P6A 6N6 Sault Ste. Marie, ON
Pault Ste. Marie, ON 5 L 3
P6A 2E6

Longueuil, PQ

Longueuil, PQ

Dryden, ON

Dryden, ON

Winnipeg, MB

Pine Falls, MB
Winnipeg, MB

Fredericton, NB

Fredericton, NB

Fredericton, NB

Torbay Bay, ND

Torbay Bay, ND

Timmins, ON

Timmins, ON

Murillo, ON

Thunder Bay, ON

Halifax, N.S

Halifax, N.S

Bridgewater, N.S.

Vernon, BC

Armstrong, $B C$
Vernon, $B C$

Riviere du Loup, PQ

Ste-Foy, $P Q$
Ste-Foy, PQ

Ottawa, ON

Kanata, ON

Port Alice, BC

Port McNeill, BC

Sherwood Park, AB Grande Prairie, $A B$

dmonton, AB

Prince Albert, SK
Prince Albert, SK

Prince Albert, SK

Smithers, BC
Smithers, BC

angton, ON

Toronto. ON

indsay, ON

Vancouver, BC Vancouver, $\mathrm{BC}$

Victoria, BC

Victoria, $\mathrm{BC}$
Victoria, $\mathrm{BC}$
$\mathrm{K} 8 \mathrm{H} 1 \mathrm{P} 4$

K8A 6W5

V2N $4 \mathrm{~N} 1$

$\mathrm{V} 2 \mathrm{~N} 4 \mathrm{~N} 1$

(604) 562.0847

25) 759-0733 $(705)$
$949-6396$
$(705)$
$949-6396$

J4H 3 Y9

J4H $3 Y 9$
J 4 H 1 E7

P8N $2 \mathrm{MB}$

P8N $2 M 8$
P9N $3 \times 3$

R3T $3 \mathrm{H} 5$

ROE 1MO

G3A $3 R_{4}$

E3B 6C2

E3B 5 P7

AOA 370

AOA 320

P4N 7S3
P4N 7S3

POT 2G0

P7E 1R8

B3J 2T9

B4V 1 K6

V1T $6 Y 5$

VOE 180
V1B 2 G9

G5R $3 Z 1$

G1V $4 C 7$
Giv $4 C 7$

K1A 1 G5

K1A $1 G 5$
K2L 2V5

VON 2 NO

VON 2 RO

VON 2 RO

T8A 2V5

TBV OA9

S6V $6 \mathrm{Z1}$

S6V $6 Z 1$

S6V 6G1

VOJ 2 NO

NOE $1 \mathrm{GO}$

M5M 1 G5

V6R $2 R 6$

V7X 1 1S7
V6B 5 A5

V8Z $1 M 5$

V8Z $1 M 5$
V8Z $1 M 5$

(506) $472-7721$ 506) $455-8602$
(506) $459-8127$

(604) $542-7830$

(613) $592-8142$

604) 956-4411

604) 956-439?

(403) 464-2875

(403) 473-5141

(306) 764-8417

(519) $875-3350$
(416) $483-8385$
(613) $589-2880$ (613) $589-2880$

(604) $962-3278$ (604) $962-3278$

(604) $847-7521$

705) $949-9461$ $705)$
$759-6774$
$(705))$
$759-6774$

(514) 679-0530 (514) 679-0530

(514) $679-5682$
$(514) 679-5682$

613) 589-2275 (613) 589-2275

(604) $962-3325$

(604) 962-3325

(705) $759-5700$

204) $945-3191$ (204) $367-2295$ (506) 458-9366 $506)$
$453-4501$
$(506)$
$452-3580$

(506) $452-3525$

(709) $772-2318$

(709) $772-2576$

807) $939-250$

(807) 939-1918

(902) 424-4321

$(902)$
(902) $543-4321$

(902) $424-7738$
$(902)$
$424-7738$

(604) $547-2111$

(604) $547-6100$

(604) $545-4411$

(418) $862-3403$ $418)$
$(418)$
$648-2219$

(819) $997-1107$ $819)$
$819)$
$997-1107$

(613) $990-3437$ $613)$
(613) $990-3437$

(604) $956-4620$

(604) $956-3843$

(604) 956-4448

(403) $427-8441$

$403)$
$539-8500$
$427-8401$

(403) $422-1457$

(306) $953-2453$

(403) $422-1457$

(306) $953-2453$

(604) $847-7504$

(416) $764-2921$

(416) $764-2921$

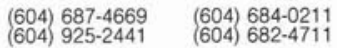

$\begin{array}{ll}\text { (604) } 474-4517 & \text { (604) } 388-0737\end{array}$

(604) $388-0737$

(604) $388-0736$

(604) 388-0775 $604)$ 388-0775
(604) $388-0775$

\section{Forest Science and Technology Board / Comité des sciences et technologies forestières}

Richard L. Rothwell, Chairman; Pierre Bernier, Vice-Chairman; Chris Lee, Secretary; lan Corns, For. Ecology; Grant Milne, For. Econ. \& Policy; Glen Blouin, For. Educ.; Errol Caldwell,
For. Pest Control; R.J. Craig. For. Harvesting; Pierre Bernier, For. Hydrol.; Crandall Benson, For. Management; Valerie LeMay, For. Measurement; J. Richardson, Silv. \& Tree Improvement; A. Harestad, Wildlife \& Fish Habitat: Michel Laverdiere, International For.; Victor Brunette, Private For.; Gary Huntley, Urban For. 